\title{
Coronary artery bypass grafting under sole Impella 5.0 support for patients with severely depressed left ventricular function
}

\author{
Shintaro Katahira ${ }^{1,2} \cdot$ Yukiharu Sugimura $^{1} \cdot$ Arash Mehdiani $^{1} \cdot$ Alexander Assmann $^{1} \cdot$ Philipp Rellecke $^{1}$. \\ Igor Tudorache ${ }^{1} \cdot$ Udo Boeken $^{1} \cdot$ Hug Aubin ${ }^{1} \cdot$ Artur Lichtenberg $^{1} \cdot$ Payam Akhyari ${ }^{1}$ (I)
}

Received: 8 March 2021 / Accepted: 18 June 2021 / Published online: 24 June 2021

(c) The Author(s) 2021

\begin{abstract}
Selection of the ideal surgical procedure for coronary revascularization in patients with severe cardiac dysfunction at times may represent a challenge. In recent years, with the advent of surgical large microaxial pumps, e.g., Impella 5.0 (Abiomed Inc., Boston, USA), specific support and effective unloading of the left ventricle has become available. In the interventional field, good results have been achieved with smaller microaxial pumps in the setting of so-called protected percutaneous coronary intervention. In this study, we would like to share our early experience with surgical coronary revascularization under the sole support of Impella 5.0, omitting the use of heart-lung machine in three cases of severe cardiac dysfunction due to complex ischemic heart disease. Effective circulatory support intraoperatively and postoperatively speaks in favor of this technique in selected patients.
\end{abstract}

Keywords Impella 5.0 · OPCAB · Low ejection fraction $\cdot$ Ischemic heart disease $\cdot$ Ischemic cardiomyopathy

\section{Introduction}

Growing evidence supports coronary artery bypass grafting (CABG) for ischemic heart disease (IHD) in front of severe cardiac dysfunction [1]. However, operative risk in this particular patient cohort with severely depressed left ventricular (LV) function remains a matter of controversy [2-4].

Off-pump CABG (OPCAB) has been advocated for the advantage of omission of cardiopulmonary bypass (CPB), which by itself is known to trigger perioperative complications, e.g., systemic inflammatory response syndrome and perioperative stroke. Moreover, OPCAB may provide certain advantage over standard CABG because of a lack of cardioplegic arrest and associated myocardial ischemia, potentially aggravating perioperative myocardial damage

Payam Akhyari

Payam.Akhyari@med.uni-duesseldorf.de

1 Department of Cardiac Surgery and Research Group for Experimental Surgery, University Hospital and Medical Faculty, Heinrich Heine University Hospital, Moorenstr. 5, 40225 Düsseldorf, Germany

2 Department of Cardiothoracic Surgery, University Hospital, Tohoku University, Sendai, Japan
[2-4]. Here, we report on a surgical method of CABG supported by Impella 5.0 (Abiomed Inc., Boston, USA) and without the use of the heart-lung machine, in the following termed Impella supported coronary artery bypass, ISCAB. We demonstrate the feasibility of this approach by sharing our experience in three successful cases in which Impella 5.0 was inserted preoperatively to stabilize hemodynamics not only during the surgery but also after surgical coronary revascularization without $\mathrm{CPB}$.

\section{Surgical procedure and perioperative management of Impella 5.0}

First, insert Impella 5.0 in the operating room prior to sternotomy. The surgical procedure for Impella 5.0 insertion has been described before. In brief, right axillary artery is exposed surgically (Fig. 1a) and a $10 \mathrm{~mm}$ graft is anastomosed in end-to-side-fashion after systemic heparinization with a targeted activated clotting time greater than $250 \mathrm{~s}$. Under combined fluoroscopy and trans-esophageal echography (TEE) control and using Seldinger technique Impella catheter is advanced via the anastomosed prosthesis and 

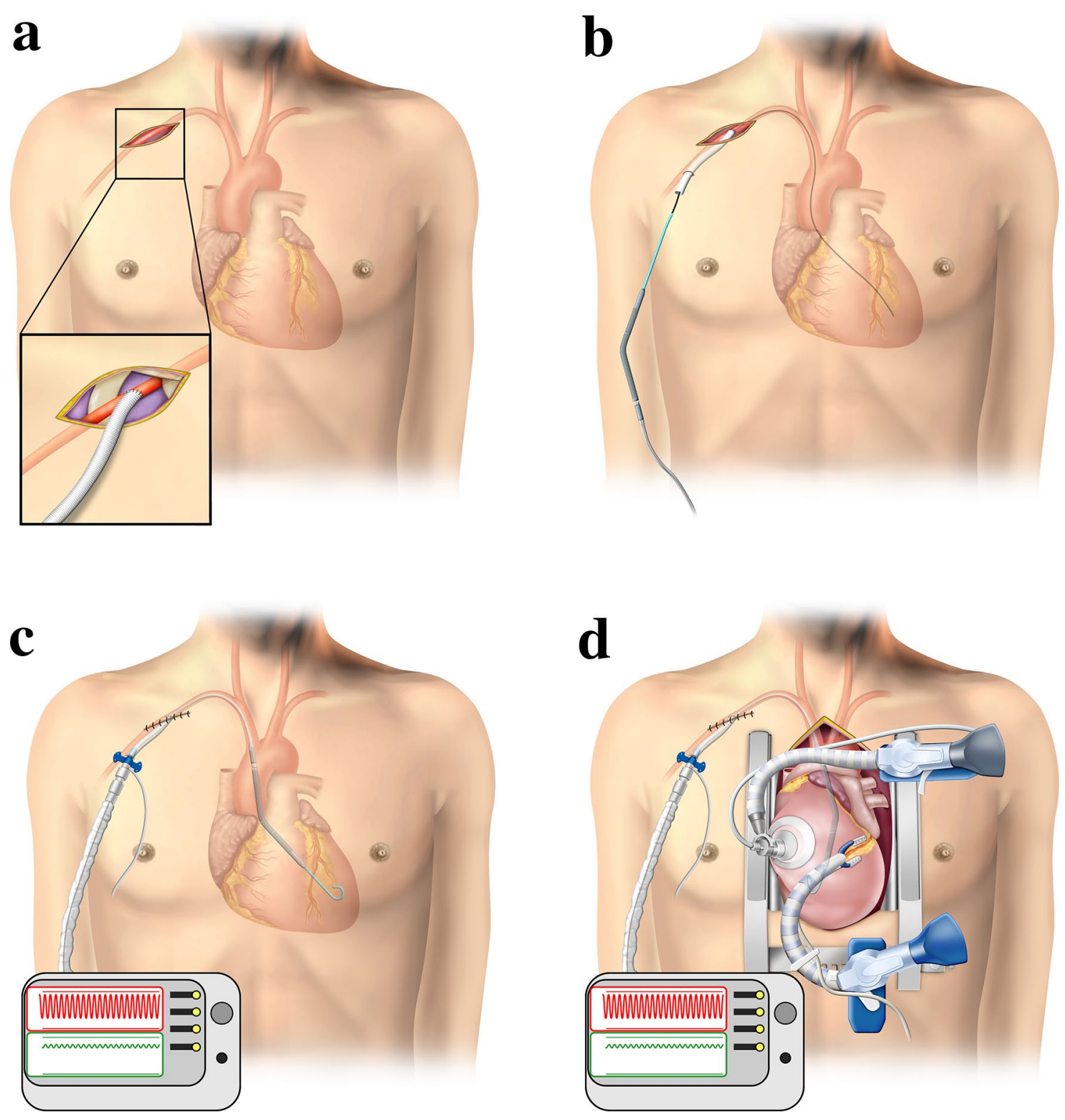

Fig. 1 Surgical procedure for off-pump coronary artery bypass grafting with Impella 5.0 support. a A skin incision was placed under the right clavicle to secure the right axillary artery. A $10 \mathrm{~mm}$ graft was anastomosed by clamping the right subclavian artery. b, c Impella 5.0 is inserted through the graft which is tunneled subcutaneously about $5 \mathrm{~cm}$ away from the infra-clavicular skin incision. $\mathbf{d}$ The access to the

positioned appropriately (Fig. 1b). Auxiliary flow and LV support are obtained.

Further operation is performed after median sternotomy and under Impella 5.0 support. (Fig. 1c) Revascularization order follows the principles of OPCAB procedure, starting with revascularization of the left anterior descending coronary artery (LAD), continuing with left circumflex coronary artery (LCX) territory and finally addressing the right coronary artery (RCA). When performing revascularization of LCX and RCA, luxation and positioning of the heart is

right subclavian artery is closed and Impella 5.0 is put into operation. Standard OPCAB via median sternotomy is performed under the support of Impella 5.0 using standard OPCAB techniques for exposure of the different coronary perfusion territories and for stabilization of the anastomosis region. $O P C A B$ off-pump coronary artery bypass grafting

promoted by the use of a stabilizer (Medtronic Octopus, additionally with Medtronic Starfish, if needed; Medtronic, Inc, Minneapolis, MN). At this stage a close monitoring of the position (TEE) and output of Impella 5.0 (displayed by the controller) is mandatory (Fig. 1d). Continuous monitoring of cardiac output and pulmonary artery pressures was performed using pulmonary artery catheter with thermodilution method, as routinely performed at our institution in all OPCAB procedures. For the instance of hemodynamic deterioration, CPB standby was provided for all of herein 
Table 1 Three cases overview

\begin{tabular}{lll} 
Case & & \\
\hline 1 & 2 & 3
\end{tabular}

Patients characteristics

\begin{tabular}{llll} 
Age (years) & 58 & 52 & 65 \\
Sex & $\mathrm{M}$ & $\mathrm{M}$ & $\mathrm{M}$ \\
Arterial hypertension & + & + & + \\
Hyperlipidemia & + & - & - \\
Diabetes & - & + & - \\
History of PCI & - & + & - \\
NYHA & 4 & 3 & 3 \\
Emergent/urgent & Emergent & Urgent & Urgent \\
Indication for CABG & 3 VD & 3 VD & LMT + 3VD \\
Operation time (min) & 353 & 381 & 431 \\
Preoperative EF (\%) & 20 & 20 & 25 \\
Postoperative EF (\%) & 30 & 30 & 29 \\
Preoperative LVEDD (mm) & 70 & 54 & 62 \\
Postoperative LVEDD (mm) & 58 & 55 & 62 \\
Preoperative RVEF (\%) & N/A & 55 & 60 \\
Preoperative RVEDD (mm) & N/A & 30 & 30 \\
Preoperative TAPSE (mm) & 17.2 & 18.5 & 19.5 \\
Preoperative TR & Trivial & 1 & Trivial \\
Impella 5.0 support duration (days) & 10 & 4 & 11 \\
\hline
\end{tabular}

$M$ male, $P C I$ percutaneous coronary intervention, $N Y H A$ New York Heart Association, $C A B G$ coronary artery bypass grafting, $3 V D$ three vessel disease, $L M T$ left main trunk, $E F$ ejection fraction, $L V E D D$ left ventricular end-diastolic diameter, $R V E F$ right ventricular ejection fraction, $R V E D D$ right ventricular end-diastolic anteroposterior diameter, TAPSE tricuspid annular plane systolic excursion, $T I$ tricuspid regurgitation presented ISCAB procedures. Moreover, intraoperative cell saver is used to support intraoperative patient blood management.

The end of the operation, Impella 5.0 support is continued for the early postoperative period, including extubation and weaning from inotropes as well as vasopressors. Intermittent evaluation by transthoracic echocardiography (TTE) or TEE serves as a crucial guiding factor to assess heart function and to decide on the optimal time point for Impella 5.0 explantation.

\section{Case presentation}

All three consecutive patients presented here fully recovered after surgery and were discharged on POD 27.0 \pm 3.46 without major adverse cardiovascular events. The total operation time was $388.3 \pm 39.5 \mathrm{~min}$, with an average of three bypasses. Table 1 shows key points of the course in these three patients. Further, the postoperative hemodynamic profile and the recovery of heart enzymes of Case 2 is shown in Fig. 2.

\section{Case 1}

A 58-year-old man was diagnosed with non-ST segment elevation myocardial infarction (NSTEMI) complicated by cardiogenic shock (CS) and was transferred to our hospital by ambulance. Coronary angiography (CAG) showed severe coronary stenosis in all three territories. TTE demonstrated
Fig. 2 Postoperative hemodynamic profile and the recovery of heart enzymes of Case 2. $C K$ creatinine kinase, $L V E F$ left ventricular ejection fraction, Troponin Ths troponin T high sensitive

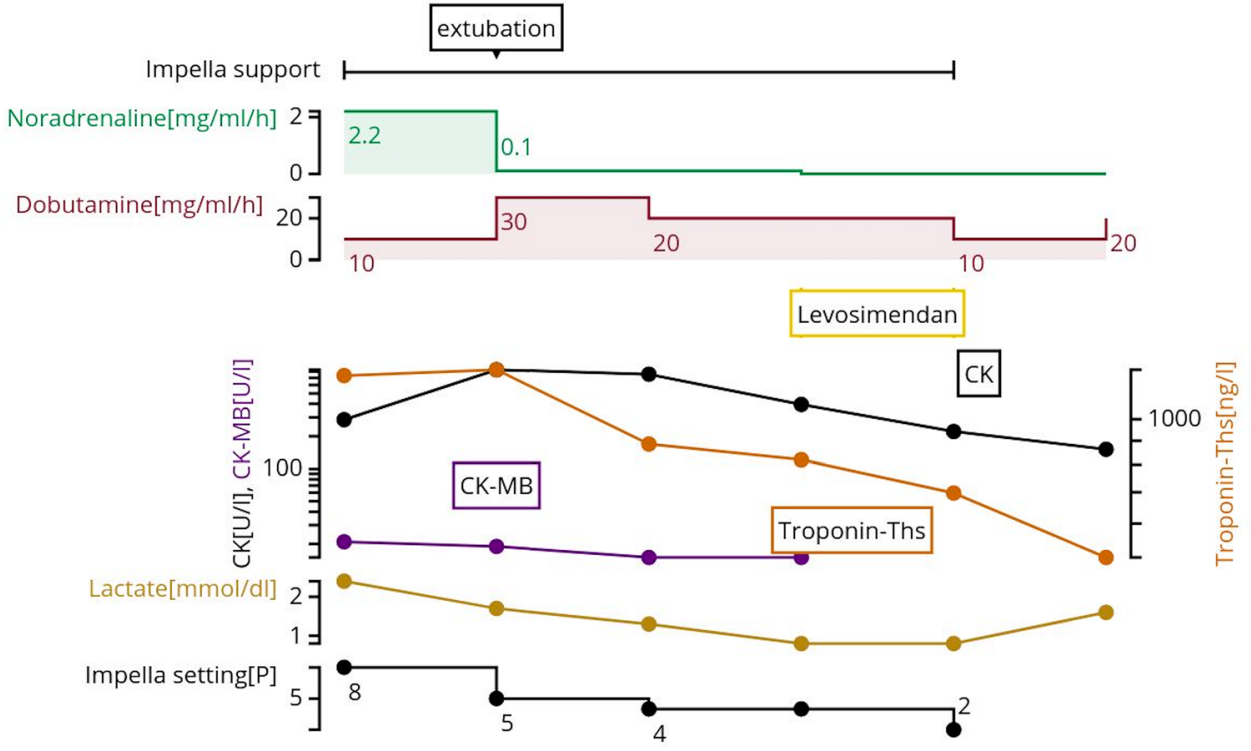

LVEF[\%] 25 
severely reduced LV ejection fraction (EF) of $20 \%$ and $\mathrm{LV}$ end-diastolic diameter (LVEDD) of $70 \mathrm{~mm}$. Impella 5.0 was inserted and coronary revascularization with four bypasses in ISCAB technique was performed: left internal thoracic artery (LITA) to LAD, saphenous vein graft (SVG) to the posterior descending branch (PD), sequential SVG to intermediate branch (IM) and further to obtuse marginal branch $(\mathrm{OM})$. Postoperative course was complicated by re-thoracotomy because of postoperative pericardial tamponade. No active bleeding site was observed after re-thoracotomy. After explantation of Impella 5.0 on 10th postoperative day (POD) the patient was transferred to cardiac rehabilitation on 31 st POD.

\section{Case 2}

A 52-year-old man was admitted on emergency basis and STEMI was confirmed. Emergency percutaneous coronary intervention (PCI) for RCA as culprit lesion was performed. 5 days later, he was introduced to our department for the purpose of left coronary artery revascularization. Severely decreased LVEF (20\%) was revealed by TTE. Since meanwhile he was not in CS, urgent revascularization was scheduled following the principles of ISCAB (LIMA-LAD, SV-OM) combined with sole Impella 5.0 support. Impella 5.0 was removed on 4th POD. The patient had a good progress and was discharged from the hospital on 25th POD.

\section{Case 3}

A 65-year-old man was admitted to another hospital because of acute heart failure (HF). CAG revealed severe three vessel disease including left main trunk lesions. After stabilization of HF, he was referred to our department for the purpose of surgery in NYHA class III. Preoperative TTE showed significantly dilated LV (LVEDD: $62 \mathrm{~mm}$ ) with severely depressed LVEF of 25\% with global hypokinesis. Urgent revascularization was performed (LIMA-LAD, SVG-OM, SVG-PD) following ISCAB technique under sole Impella 5.0 support. Postoperative course was complicated by viral pneumonia, however, Impella 5.0 was successfully weaned on 11th POD and he was transferred for rehabilitation on 25th POD.

\section{Discussion}

Impella 5.0 is indicated for patients with circulatory failure refractory to conservative therapy options [5-7]. Most recent literature in the field of HF management suggests an increasing use of large microaxial pumps as bridging therapy or as a temporary perioperative or peri-procedural support. Impella 5.0 is able to effectively unload
$\mathrm{LV}$, thereby reducing wall stress and myocardial oxygen consumption [8]. In the setting of low cardiac function or CS good results have been obtained for Impella supported PCI [9]. However, the value of perioperative Impella support for high-risk CABG in patients with severely reduced $\mathrm{LV}$ function is yet to be determined.

Current evidence speaks in favor of CABG for IHD with LV dysfunction, but the appropriate operative method is still controversial [1]. A recent comparison between onpump CABG and OPCAB for coronary revascularization in patients with $\mathrm{LV}$ dysfunction has demonstrated a reduction of postoperative complications by OPCAB [3]. On the other hand, in randomized controlled trials $\mathrm{OPCAB}$ has been associated with lower numbers of grafts and incomplete revascularization, which may have a negative effect on long-term results of surgical revascularization [4]. In present series, despite severely impaired LV function complete revascularization has become feasible after stabilization of hemodynamics by implantation of Impella 5.0 prior to surgical revascularization on beating heart without the use of $\mathrm{CPB}$. As this procedure employs a microaxial blood pump but yet avoids the use of CPB, we termed this procedure Impella supported $\mathrm{CAB}$ (ISCAB). In addition, Impella 5.0 was able to operate without any problem even during luxation of the heart for revascularization of the LCX or RCA perfusion territory. Since LV was unloaded, the surgical field for anastomosis could be secured, providing certainly a wider range of freedom than in comparable cases with LV dilatation and reduced EF. Although the LCX anastomosis was performed in a stable hemodynamics, the precise performance and variations in circulatory support by Impella 5.0 at the time of elevating apex remains yet to be evaluated in larger patients' cohorts. Further, the possibility of injury to cardiac structures during maneuvers of cardiac positioning for better exposure of the LCX and RCA perfusion territory warrants further evaluation of this technique, meanwhile it is considered necessary to perform any elevation maneuvers carefully and under repetitive TEE-control of the position of Impella tip [10].

Another advantage of using Impella 5.0 lies in its usefulness as a ventricular support device not only in the preand intra-operative time, but also in the early postoperative period. IABP (Intra-Aortic Balloon Pumping) and venoarterial extracorporeal membrane oxygenation (VA-ECMO) have also been used for temporary support after CABG in high-risk patients, however, both systems have shown considerable limitations when used in patients with severe LV dysfunction, due to problems with the limited maximal assist flow rate or due to detrimental increase in LV afterload, respectively [5-7]. In contrast, Impella 5.0 is highly effective in unloading $\mathrm{LV}$ while supporting peripheral circulation and preventing organ failure caused by low output syndrome 
[11]. In fact, in our case series, despite the preoperatively severe cardiac dysfunction, all patients recovered without deterioration of organ function.

Additionally, in the case of such severely impaired LV function, on-pump beating CABG with CPB may present an alternative. However, in that case, VA-ECMO, IABP or both may become necessary after surgery. Therefore, and also from an economic point of view, it was considered better to use only Impella 5.0.

We were able to obtain good results by performing ISCAB, i.e., CAB under Impella 5.0 support, for IHD with severe cardiac dysfunction. Some previous articles support our favorable result of ISCAB for patients with severely impaired LVEF [12, 13]. With sufficient monitoring when elevating the heart apex, this strategy is considered to be an option for patients with severe cardiac dysfunction to perform facilitated surgical revascularization with omission of heart-lung machine and protected hemodynamic stability in the perioperative course.

Supplementary Information The online version contains supplementary material available at https://doi.org/10.1007/s10047-021-01285-1.

Funding Open Access funding enabled and organized by Projekt DEAL. The authors received no specific funding for this work.

\section{Declarations}

Conflict of interest The authors do not have any conflicts of interest.

Open Access This article is licensed under a Creative Commons Attribution 4.0 International License, which permits use, sharing, adaptation, distribution and reproduction in any medium or format, as long as you give appropriate credit to the original author(s) and the source, provide a link to the Creative Commons licence, and indicate if changes were made. The images or other third party material in this article are included in the article's Creative Commons licence, unless indicated otherwise in a credit line to the material. If material is not included in the article's Creative Commons licence and your intended use is not permitted by statutory regulation or exceeds the permitted use, you will need to obtain permission directly from the copyright holder. To view a copy of this licence, visit http://creativecommons.org/licenses/by/4.0/.

\section{References}

1. Berger PB, Velianou JL, Aslanidou Vlachos H, et al. Survival following coronary angioplasty versus coronary artery bypass surgery in anatomic subsets in which coronary artery bypass surgery improves survival compared with medical therapy. Results from the bypass angioplasty revascularization investigation (BARI). J Am Coll Cardiol. 2001;38:1440-9. https://doi.org/10.1016/s07351097(01)01571-6.
2. Guan Z, Guan X, Gu K, et al. Short-term outcomes of on- vs off-pump coronary artery bypass grafting in patients with left ventricular dysfunction: a systematic review and meta-analysis. J Cardiothorac Surg. 2020;15:84. https://doi.org/10.1186/ s13019-020-01115-0.

3. Keeling WB, Williams ML, Slaughter MS, et al. Off-pump and on-pump coronary revascularization in patients with low ejection fraction: a report from the society of thoracic surgeons national database. Ann Thorac Surg. 2013;96:83-8. https://doi.org/10. 1016/j.athoracsur.2013.03.098 (discussion 88-89).

4. Jarral OA, Saso S, Athanasiou T. Off-pump coronary artery bypass in patients with left ventricular dysfunction: a meta-analysis. Ann Thorac Surg. 2011;92:1686-94. https://doi.org/10.1016/j.athor acsur.2011.06.006.

5. Vallabhajosyula S, O'Horo JC, Antharam P, et al. Venoarterial extracorporeal membrane oxygenation with concomitant impella versus venoarterial extracorporeal membrane oxygenation for cardiogenic shock. ASAIO J. 2020;66:497-503. https://doi.org/ 10.1097/MAT.0000000000001039.

6. Patel SM, Lipinski J, Al-Kindi SG, et al. Simultaneous venoarterial extracorporeal membrane oxygenation and percutaneous left ventricular decompression therapy with impella is associated with improved outcomes in refractory cardiogenic shock. ASAIO J. 2019;65:21-8. https://doi.org/10.1097/MAT.0000000000000767.

7. Pappalardo F, Schulte C, Pieri M, et al. Concomitant implantation of Impella((R)) on top of veno-arterial extracorporeal membrane oxygenation may improve survival of patients with cardiogenic shock. Eur J Heart Fail. 2017;19:404-41. https://doi.org/10.1002/ ejhf.668.

8. Donker DW, Brodie D, Henriques JPS, et al. Left ventricular unloading during veno-arterial ECMO: a review of percutaneous and surgical unloading interventions. Perfusion. 2019;34:98-105. https://doi.org/10.1177/0267659118794112.

9. O'Neill WW, Schreiber T, Wohns DH, et al. The current use of Impella 2.5 in acute myocardial infarction complicated by cardiogenic shock: results from the USpella Registry. J Interv Cardiol. 2014;27:1-11. https://doi.org/10.1111/joic. 12080.

10. de Armas SIA, Kumar S, Almustafa A, et al. Left ventricular rupture after Impella $(\mathrm{R})$ placement during high risk percutaneous coronary intervention. Cardiovasc Revasc Med. 2019. https://doi. org/10.1016/j.carrev.2019.06.011.

11. Tongers J, Sieweke JT, Kuhn C, et al. Early escalation of mechanical circulatory support stabilizes and potentially rescues patients in refractory cardiogenic shock. Circ Heart Fail. 2020;13: e005853. https://doi.org/10.1161/CIRCHEARTFAILURE.118. 005853.

12. Takahashi K, Nakata J, Kurita J, et al. Impella-assisted coronary artery bypass grafting for acute myocardial infarction. Asian Cardiovasc Thorac Ann. 2020;28:115-7. https://doi.org/10.1177/ 0218492319888053.

13. Pepino P, Coronella G, Oliviero P, et al. Successful use of the Impella recover LP 5.0 device for circulatory support during off-pump coronary artery bypass grafting. Int J Surg Case Rep. 2014;5:803-5. https://doi.org/10.1016/j.ijscr.2014.07.013.

Publisher's Note Springer Nature remains neutral with regard to jurisdictional claims in published maps and institutional affiliations. 